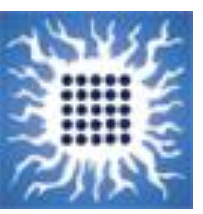

Институт за нуклеарне науке „Винча“

Универзитет у Београду

VINČA Institute of Nuclear Sciences

University of Belgrade

2019

Improved single-step extraction performance of aqueous biphasic systems using novel symmetric ionic liquids for the decolorisation of toxic dye effluents

Dimitrijević, Aleksandra; Jocić, Ana; Zec, Nebojša; Tot, Aleksandar; Papović, Snežana; Gadžurić, Slobodan; Vraneš, Milan; Trtić-Petrović, Tatjana M.

Published in:

Journal of Industrial and Engineering Chemistry

DOI:

10.1016/j.jiec.2019.04.017

Document version:

Peer reviewed article (often known as postprint article)

Recommended citation:

Dimitrijević, A., Jocić, A., Zec, N., Tot, A., Papović, S., Gadžurić, S., ... \& Trtić-Petrović, T. (2019). Improved single-step extraction performance of aqueous biphasic systems using novel symmetric ionic liquids for the decolorisation of toxic dye effluents. Journal of Industrial and Engineering Chemistry, 76, 500-507. 


\section{Improved single-step extraction performance of aqueous biphasic systems using novel symmetric ionic liquids for the decolorisation of toxic dye effluents}

Aleksandra Dimitrijević, ${ }^{\mathrm{a}}$ Ana Jocić, ${ }^{\mathrm{a}}$ Nebojša Zec, ${ }^{\mathrm{b} 1}$ Aleksandar Tot, ${ }^{\mathrm{b}}$ Snežana Papović, ${ }^{\mathrm{b}}$ Slobodan Gadžurić, ${ }^{\mathrm{b}}$ Milan Vraneš ${ }^{\mathrm{b}}$ and Tatjana Trtić-Petrovićc ${ }^{\mathrm{a}}{ }^{*}$

${ }^{a}$ Laboratory of Physics, Vinča Institute of Nuclear Sciences, University of Belgrade, 11001 Belgrade,Serbia; daleksandra@vinca.rs,ana.jocic@vinca.rs,ttrtic@vinca.rs ${ }^{b}$ Faculty of Sciences, University of Novi Sad, Department of Chemistry, Biochemistry and Environmental Protection, 21000 Novi Sad, Serbia; aleksandar.tot@dh.uns.ac.rs, snezana.papovic@dh.uns.ac.rs,slobodan.gadzuric@dh.uns.ac.rs,milan.vranes@dh.uns.ac.rs

*Corresponding author:

Tatjana Trtić-Petrović

Laboratory of Physics

Vinča Institute of Nuclear Sciences

University of Belgrade

P.O. Box 522, 11001 Belgrade, Serbia

Tel: +381116447700

ttrtic@vinca.rs

Graphical abstract

\footnotetext{
${ }^{1}$ Present address: German Engineering Materials Science Centre (GEMS) at Heinz MaierLeibnitz Zentrum (MLZ), Helmholtz-Zentrum Geesthacht GmbH, München, Germany; Nebojsa.Zec@hzg.de
} 


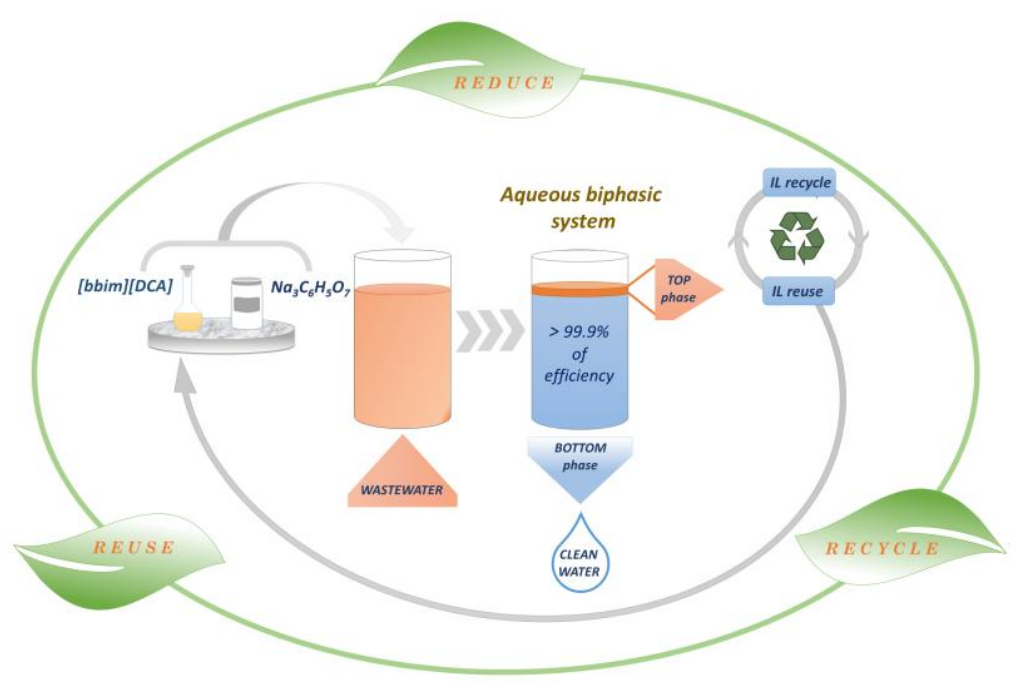

\section{Highlights}

- New symmetric ILs were used for extraction of widely used toxic textile dyes from waste water;

- Low amount of eco-friendly citrate salt was applied to generate ABS;

- Efficiency of $99.4 \%$ for removal of Orange II in a single step extraction was achieved;

- Maximal efficiency was obtained using minimal amounts of ABS ingredients;

- Recycling procedure of applied ILs was successfully tested and proposed.

\section{$\dagger$ ESI}

\section{Abstract}

The novel aqueous biphasic systems based on symmetric imidazolium ionic liquids (ILABSs) for the extraction of toxic and resistive dyes from water was studied. The cation symmetricity of ILs strongly influence the outstanding ability of 1,3-dibutylimidazolium dicyanamide, [bbim][dca], to generate ABS with the exceptionally low amount of ecofriendly citrate salt. A remarkable potential of [bbim][dca]/citrate based ABS to remove orange II from wastewater with concentration up to 1000 fold and efficiency above $99.4 \%$ in a single step was achieved. The improved safety of proposed method nominates it as promising future strategy for pollution prevention.

Keywords: Aqueous biphasic system; binding energy; extraction; symmetric ionic liquid; synthetic dyes; wastewater. 


\section{Introduction}

Implementing Green Chemistry philosophy based on twelve guiding principles [1] in dye related industries is a challenging task for both scientists and manufacturers. The structures of synthetic dyes extensively used in textile, dyeing, paper, printing, color photography, pharmaceutical, food, cosmetics, and other industries, are based on complex aromatic hydrocarbons which make them highly stable and non-biodegradable [2,3]. The most of artificial dyes and their metabolites are highly toxic, carcinogenic and largely affect the photosynthetic activity [4]. Tons of synthetic dyes are produced annually at a global scale, and approximately $15 \%$ of them are discharged only by textile industries into the hydrosphere [5]. Taking into account the scope of textile industry and a tremendous amount of water it consumes annually adding the toxic chemicals to waste streams, it is considered as one of the most ecologically harmful industry in the world [6]. Many parts of the world are already suffering from medium to high levels of water stress, and the situation is more critical if we take into account anticipation that consumption of water by dye-related industries will increase for $50 \%$ by 2030 [7].

Azo dyes represent the most widespread class of dyes constituting more than $70 \%$ of the global industrial demand ( $\sim 9$ million tons) [8]. Azocolorants by means of reductive cleavage of azo groups may release 22 highly carcinogenic aromatic amines [9] and for that reason are banned in the European Union (Annex XVII of the REACH regulation; No. 1907/2006) [10]. From 896 azo dyes in the database with known chemical structure, 426 of them $(48 \%)$ are potential precursors for 22 regulated toxic aromatic amines [11]. Anthraquinones are the second most important group which complement azo dyes in providing the whole shade gamut [12] and all the important natural red dyes in contrast to the azocolorants which have no natural counterparts. Both natural and synthetic anthraquinones have been employed as colorants in food, drugs, cosmetics, hair dyes, gasoline, and textiles. Many studies confirmed their mutagenicity, allergenicity and carcinogenicity affecting thus the aquatic biota and human health $[13,14]$.

The treatment of contaminated wastewaters is of the highest importance in order to prevent harmful impact on aquatic ecosystems as a result of direct exposure to synthetic dyes. Various methods (filtration, coagulation, precipitation, adsorption, ion exchange, microbiological or enzymatic decomposition and oxidation) have been evaluated for the removal of the dyes from wastewaters [3, 15-20]. The most of these processes display drawbacks such as high-running cost, low efficiency, a labor-intensive operation, 
consumption of a large amount of volatile organic compounds and operate under high or low temperatures. Undoubtedly, the best method to protect the environment is to prevent pollution through substitution of the toxic chemicals, process modification and, equipment upgrade, water reuse and product redesign. However, before implementing new strategies, which will require years at the best, the urgent action must be taken in development of simple, efficient and low-cost methods in order to repair the damage to water issues.

Two commonly used water-soluble textile dyes, Orange II (OII) and Remazol Brilliant Blue R (RBBR) also known as Cavalite Brilliant Blue R or Reactive Blue 19 (Fig. 1), have been selected in this work as a model dyes in order to examine new proposed separation method for dyes removal from industrial wastewaters. According to their chromophores, OII and RBBR are classified as azo and anthraquinone dyes, respectively. Both dyes, very toxic and carcinogenic, are widely used in the textile industry, and their release into the natural environment is undesirable because of the adverse effect on aquatic life and destructive consequence for ecosystems [15].

As the new extractants and green alternatives to conventional solvents, newly synthesized ionic liquids (ILs) were used in this work. The definition of the ionic liquids and their remarkable properties are widely known and discussed [21-23]. By using different ions it is possible to design ILs to fit the requirements of a certain application which is the most important feature of ILs [24]. Liquid-liquid extraction based on water-immiscible ILs was already investigated for the extraction of the textile dyes from wastewater [25]. However, the acquired efficiencies were not good enough, and the implementation of extraction technique based on hydrophobic ILs on a large scale would be limited due to high costs, since these types of ILs often contain expensive and non-stable fluorinated anion [25]. On the other side, hydrophilic ILs have much greater potential to be applied in separation processes due to the availability of "greener" anions and less expensive materials [26].

Aqueous biphasic systems (ABSs) based on ionic liquids (IL-ABSs) are environmentally safer option to traditional liquid-liquid extraction and offers the possibility to take advantage of water-miscible ILs. IL-ABS consists of two macroscopic liquid phases, typically formed by different pairs of aqueous solutions (polymer-polymer, polymer-salt or salt-salt) at appropriate concentrations [27]. Recent studies have shown the outstanding performance of IL-ABSs in the selective separation and extraction processes for a wide range of value-added compounds, biomolecules, pesticides, metals etc. [28-30]. Moreover, IL-ABSs have significant advantages such as the aqueous nature of the coexisting phases, fast phases separation, low cost and simple scale-up to be used in continuous operation [26, 29]. Due to 
these reasons IL-ABSs, as a relatively novel separation method, have been an object of growing interest. The extraction capability of ABS is strongly linked with physicochemical properties of the phases which can be easily manipulated by adequate selection of IL, saltingout agent and composition [31-34].

In this work, we proposed the use of ABS based on new ILs with equal alkyl substituents in both, N1 and N3, positions of imidazolium cation (referred as symmetric ILs) as a removal strategy for synthetic dyes from wastewater. Initially, the phase diagrams of IL-ABSs were determined to gather insight into their formation aptitude, and then the ability of IL-ABS platform to extract the dye pollutants from water was investigated. Further, the optimization of the procedure was performed in order to demonstrate and assure the process feasibility. Using the molecular dynamics simulations an outstanding extraction performance of new ILABS is discussed.

\section{Experimental}

\section{Materials}

1-ethylimidazole, 1-butylimidazole, 1-bromoethane, 1-bromobutane, 1-bromohexane (mass fraction purity, $\omega \geq 0.98)$, sodium dicyanamide $\left(\mathrm{NaC}_{2} \mathrm{~N}_{3}\right)$, acetone, potassium phosphate $\left(\mathrm{K}_{3} \mathrm{PO}_{4}\right)$, and sodium citrate $\left(\mathrm{Na}_{3} \mathrm{C}_{6} \mathrm{H}_{5} \mathrm{O}_{7}\right)$ (p.a. purity) were purchased from Sigma Aldrich (St. Louis, MO, USA). Textile dyes: Orange II (4-(2-hHydroxy-1naphthylazo)benzenesulfonic acid sodium salt) and Remazol brilliant blue R (1-amino-9,10dioxo-4-[3-(2-sulfonatooxyethylsulfonyl)anilino]anthracene-2-sulfonate disodium salt) were obtained from Acros Organics (New Jersey, USA).

Four symmetric ILs were synthesized in this work (Table 1). 1,3-diethylimidazolium bromide ([eeim][Br] ) and 1,3-dibutylimidazolium bromide ([bbim][Br]) were prepared by microwave-assisted procedure using a single mode microwave reactor Discover SP (CEM Corporation, Matthews, USA) [35]. Briefly, the equimolar amounts of either 1-ethylimidazole and 1-bromoethane or 1-butylimidazole and 1-bromobutane were mixed in a quartz vessel and irradiated at $240 \mathrm{~W}$ and heated either at $353.15 \mathrm{~K}$ for $7 \mathrm{~min}$ or at $393.15 \mathrm{~K}$ for $15 \mathrm{~min}$, respectively. Obtained colorless liquids were dried and kept in desiccator under $\mathrm{P}_{2} \mathrm{O}_{5}$.

The dicyanamide based ILs, 1,3-diethylimidazolium dicyanamide ([eeim][dca]), 1,3dibutylimidazolium dicyanamide ([bbim][dca]) and 1-hexyl-3-ethylimidazoliumdicyanamide ([heim][dca]) were synthesized using corresponding ILs with bromide ions. The equimolar amounts of IL ([eeim][Br], [bbim] $[\mathrm{Br}]$ and [heim][Br]) and $\mathrm{NaC}_{2} \mathrm{~N}_{3}$ were dissolved in 
acetone. The precipitated sodium bromide was removed by filtration, and acetone was evaporated at $343.15 \mathrm{~K}$ under decreased pressure. The chemical structures of the synthesized ILs were determined by NMR spectroscopy and obtained spectra together with peak assignation are given in Figs. S1a-S1d and Table S1a-S1d $\dagger$, respectively. The purities of the synthesized ILs are also presented in Table 1.

\section{Phase diagrams and tie-lines (TLs)}

The biphasic phase diagrams were determined applying the cloud point titration method at room temperature $(296.15 \pm 1 \mathrm{~K})$ and atmospheric pressure $(0.1 \mathrm{MPa})$ [33, 36-38]. The aqueous solution of inorganic salt $(\omega=50 \%)$ was added drop by drop to the aqueous solution of IL $(\omega=60 \%)$ of known mass until the mixture become cloudy. After the addition of the salt solution, the mass of the mixture was recorded using an analytical balance (CP224S, Sartorius, Germany) with an uncertainty of $\pm 10^{-4} \mathrm{~g}$. Then, water was added until the mixture becomes clear and the system was weighted again. The mixture was shaken after each addition of solution using a vortex agitator (Reax Top, Heidolph, Germany) at $2500 \mathrm{rpm}$. This procedure was repeated as far as enough points were obtained for the construction of the binodal curve. The experimental solubility data of the biphasic phase diagrams were fitted using empirical Merchuk equation (Eq. S1 †) [39].

The tie-lines (TLs) which connect two nodes on the binodal curve were determined by a gravimetric method and calculated by application of lever arm rule [39] (detailed procedure was given in the ESI).

\section{Extraction of the dyes by ABS}

The extraction performance of proposed IL-ABSs was evaluated using a mixture of $30 \%$ of IL, $10 \%$ of $\mathrm{K}_{3} \mathrm{PO}_{4}$ and $60 \%$ of aqueous solution of dye $\left(1 \mathrm{~g} \mathrm{dm}^{-3}\right)$. For the optimization studies, a different ternary mixture compositions $\left\{\left[\right.\right.$ bbim] $[$ dca $]+\mathrm{Na}_{3} \mathrm{C}_{6} \mathrm{H}_{5} \mathrm{O}_{7}+$ aqueous solution of dye \} were prepared in order to investigate the influence of TLL on the distribution ratio of OII. For the screening of improved extraction efficiencies along the selected TL the ternary systems $\left\{[\right.$ bbim $][$ dca $]+\mathrm{Na}_{3} \mathrm{C}_{6} \mathrm{H}_{5} \mathrm{O}_{7}+$ aqueous solution of dye $\}$ were prepared at different mixture compositions and different mass ratios (mass of water/mass of the IL-rich phase). All prepared solutions were vigorously vortex-stirred for $2 \mathrm{~min}$ at $2500 \mathrm{rpm}$ and left to equilibrate for $2 \mathrm{~h}$ allowing the complete partitioning of dyes between the two phases. After 
the separation of the phases, the dyes were quantified by UV spectrometry (UV-2600 Spectrophotometer, Shimadzu, Japan) at a wavelength of $485 \mathrm{~nm}$ for OII and $592 \mathrm{~nm}$ for RBBR. In order to avoid the interference of ABS phase constituents in the quantification, blank control samples were always used. All experiments were performed in triplicates, and the mean values were shown.

\section{Computational details}

The geometry optimization was conducted using density functional theory (DFT) and Jaguar 9.0 program as implemented in Schrödinger Materials Science Suite 2015-4. For DFT calculations AMBER force field was used, and intermolecular non-covalent interactions (NCI) were investigated [40, 41]. Geometry optimizations were followed by harmonic frequency analysis to ensure that the obtained structures were true minima due to the absence of imaginary wavenumbers.

Based on DFT calculations, ion-pair binding energies between cation and anion were calculated. To avoid a basis-set superposition error (BSSE) binding energies $\left(\Delta \mathrm{G}_{\text {bin }}\right)$ were counter-poise corrected [41]. The final expression for the ion-pair binding energy was as follows:

$$
\Delta G_{\mathrm{bin}}=E_{\mathrm{cp}}(\mathrm{C}-\mathrm{A})-E_{\min }(\mathrm{C})-E_{\min }(\mathrm{A})+\Delta Z P V E
$$

where $E_{\mathrm{CP}}(\mathrm{C}-\mathrm{A})$ is the counter-poise corrected electronic energy of the ion pair and $E_{\min }(\mathrm{C})$ and $E_{\min }(\mathrm{A})$ are the electronic energies of the cation and the anion in their minimum-energy geometries. Zero-point vibrational energies $(\triangle Z P V E)$ were calculated using scaled B3LYP/6$31+\mathrm{G}(\mathrm{d})$ vibrational frequencies.

The MD simulations were performed using the DL-POLY program in order to obtain realistic binding energy. For simulations, the AMBER force field was used and the LJ parameters and the partial charge were assigned from geometrical optimization of investigated ILs and dyes. The simulations were conducted in the canonical ensemble using the NoseHoover extended system method. Periodic cubic boundary conditions were employed. Longrange electrostatic interactions were computed via the Ewald method with a conducting surrounding medium, resulting in essentially no truncation of these interactions. The trajectories were integrated via the Verlet leapfrog algorithm combined with the quaternion method for rotations with a time step of 2 fs. Equilibrium simulations were carried out with 10 ns equilibration, followed by a $10 \mathrm{~ns}$ trajectory from which averages are computed.

\section{Results and discussion}




\section{Phase diagrams of the ABSs and binding energies}

The new phase diagrams of the ABSs based on the symmetric imidazolium ILs combined with phosphate salt were determined in this work. Potassium phosphate was selected in order to characterize and compare phase diagrams and the extraction parameters of IL-ABSs with different hydrophobicity [42]. The solubility curves which belong to new IL-ABSs \{IL + $\left.\mathrm{K}_{3} \mathrm{PO}_{4}+\mathrm{H}_{2} \mathrm{O}\right\}$ are given in Fig. 2 and obtained parameters tabulated in Tables $\mathrm{S} 2$ and $\mathrm{S} 3 \uparrow$. The experimental data were fitted by empirical Merchuk equation (Eq. S1 †) [39] and the regression parameters for this equation were estimated by the least-squares numerical method (Table S4 $\dagger$ ). The values of $R^{2} \geq 0.9971$ and low values of $\sigma$ demonstrate that Merchuk equation is suitable to fit the experimental data.

The two-phase region is localized above the solubility curve while the monophasic region is presented below and in general, the larger the biphasic region, the higher is the ability of the IL to undergo liquid-liquid separation and to form an ABS. Hydrophobicity range of the studied ILs is very wide and cover the extensive region of the biphasic diagram (Fig. 2), from the most hydrophobic IL ([bbim][dca]) to very hydrophilic IL ([eeim][Br]). It is clear from Fig. 2 that the longer alkyl chains on imidazolium cation enhance the hydrophobicity of ILs which decreases their affinity for water and implies better ability to form ABS. If the concentration of IL is fixed at $1 \mathrm{~mol} \mathrm{dm}^{-3}$ (Fig. 2), the required minimal molar concentration of $\mathrm{K}_{3} \mathrm{PO}_{4}$ to induce cloudy solution increases in the following order: $0.093 \mathrm{~mol} \mathrm{dm}^{-3}$ $([$ bbim $][$ dca $])<0.543 \mathrm{~mol} \mathrm{dm}^{-3}\left([\right.$ bbim] $[\mathrm{Br}])<0.725 \mathrm{~mol} \mathrm{dm}^{-3}([$ eeim] $[$ dca $])<0.944 \mathrm{~mol}$ $\mathrm{dm}^{-3}([$ eeim $][\mathrm{Br}])$.

The results agree with previous studies which address the effect of IL chemical structures on the ABS formation aptitude [33]. The abilities of ILs with [dca] ${ }^{-}$to form ABS are significantly higher than $[\mathrm{Br}]^{-}$based ILs due to its more hydrophobic nature and lower affinity to form hydrogen bonds.-The ABS based on [bbim][dca] exhibits the largest biphasic area, and its solubility curve is the closest to origin indicating that exceptionally low amount of salt $(\sim 2 \%)$ is required to induce two phases, facilitating thus the use of the ABS for onestep extraction and enrichment of an analyte. ILs containing [eeim] cation pronounce hydrophilic character indicating hard competition with inorganic salt for water molecules. Consequently, two-phase areas of ABS based on [eeim] ${ }^{+}$are smaller and more salt is necessary to salt them out in comparison with [bbim $]^{+}$.

The spatial arrangement of side alkyl-chains on imidazolium cation has the significant influence on the ABS formation [43]. In order to evaluate the influence of cation symmetry on 
the solubility curves position, different ILs with the same total number of substituent methyl groups (structural isomers: [bbim] and [heim]) were compared (Fig. 2 (Inset)). It is obvious that [bbim][dca] has a significantly higher ability to induce ABS than its structural isomer [heim][dca]. On the other hand, the proximity of binodal curves which correspond to [bbim] $[\mathrm{Br}]$ and $[\mathrm{heim}][\mathrm{Br}]$ suggests that symmetry/asymmetry of imidazolium cation impact the ABS formation and alkyl chains from both positions contribute to the overall hydrophobicity of IL. That contribution is much more pronounced in the case of stronger hydrogen bond acceptor such as [dca].

The relative cation-anion strength (binding energies, $\Delta G_{\mathrm{bin}}$ ) for the studied ILs were calculated and plotted in Figure 3. $\Delta G_{\text {bin }}$ are connected with the structural features of the ILs such as the cation structure and ring size, aromaticity, alkyl chain length, anion nature and size, and ion charge densities [44]. Although cation-anion interactions in ILs are mainly dominated by Coulombic forces, the hydrogen bonding, polar and dispersive interactions also play a significant role $[32,44]$. The more negative values of $\Delta G_{\text {bin }}$ indicate stronger interactions between cation and anion e.g. the lowest $\Delta G_{\text {bin }}([$ eeim $][\mathrm{Br}])$ indicates the strongest interactions between constituents of IL and consequently the lowest ability for ABS formation.

Although [heim][dca] has the lowest $\Delta G_{\text {bin }}$ value, it is better solvated by water molecules than symmetric IL with the equal total number of carbon atoms in the side alkyl chains ([bbim][dca]) (Fig. 2). This leads to the conclusion that other factors such as the contributions of alkyl chains on imidazolium cation to the total hydrophobicity of IL and the steric effects must be taken into account in order to discuss IL affinity for water. Long hexyl chain on the $\mathrm{N} 1$ position (hydrophobic moiety) and asymmetry of [heim $]^{+}$are the main reasons to induce weak cation-anion interactions. Many studies confirmed that the increase of the alkyl chain length generally leads to a strength decrease of the Coulombic and polar forces, while at the same time the dispersive interactions between IL ions are increasing. [45, 46]. Ethyl group on the N3 position of imidazole ring contribute to better solvation and can be referred to as a hydrophilic moiety of $[\text { heim }]^{+}$. On the other side, $[\text {bbim }]^{+}$cation is symmetrical which explain a more negative $\Delta G_{\text {bin }}$, but two hydrophobic moieties (butyl groups) cause weaker ion interactions with water molecules. Additionally binding energies are more negative for bromide based ILs in comparison with dicyanamide ILs with the same cation, which is expected, since $\mathrm{Br}^{-}$is smaller anion and it can come closer to IL cation forming stronger Coulombic forces. 


\section{Extraction of the targeted textile dyes}

TLs are necessary and exceptionally valuable from the aspect of designing and optimization of the extraction in order to achieve high removal efficiencies of dye pollutants from wastewater. Fig. S2 and Table S5 † show TLs for novel IL-ABSs. ABS based on [bbim][dca] and $\mathrm{K}_{3} \mathrm{PO}_{4}$ may contain up to $80 \%$ of water. This notable behavior is of the highest importance for "greenness" of the extraction process and it is exceptionally worth to note that the system can be tailored so that the salt-rich phase of the ABS contains over $90 \%$ of water.

A fixed ternary mixture composition $\left\{30 \% \mathrm{IL}+10 \% \mathrm{~K}_{3} \mathrm{PO}_{4}+60 \%\right.$ aqueous dye solution $\left.\left(1 \mathrm{~g} \mathrm{dm}^{-3}\right)\right\}$ was chosen to compare the ability of the studied ILs to extract two structurally different dyes from an aqueous sample. Given that concentrations of ABS components and $\mathrm{pH}$ value were kept constants, the dye partitioning was only influenced by their intrinsic properties. In all investigated ABSs, the dyes preferentially migrated into the upper IL-rich phase (Fig. 4a). Obtained distribution ratios (D) are ranged between 218 and 394 for RBBR, and from 345 to 703 for OII. From Fig. 4b it can be seen that D for both dyes decrease in the following order $[$ bbim $][$ dca $]>[$ bbim $][\mathrm{Br}]>[$ eeim $][\mathrm{dca}]>[$ eeim $][\mathrm{Br}]$. The extraction efficiency above $97 \%$ was achieved for all investigated ABSs and follows the same order. The results indicate that both IL constituents (cation and anion) have the influence on the extraction behavior.

The $\mathrm{pH}$ value of the ABS mixtures is around 12, as a result of high potassium phosphate content, and both dyes are presented in an anion form at this $\mathrm{pH}$ according to their $\mathrm{p} K_{\mathrm{a}}$ values. The detailed $\mathrm{pH}$ data and speciation curves for OII and RBBR are given in Fig. S3 †. Both compounds are hydrophilic displaying very low distribution coefficient in the octanol/water system ( 0.033 and 0.001 for OII and RBBR, respectively) indicating thus low extraction efficiency.

The driving forces which govern the partition behavior of selected dyes are the hydrophobicity of the ILs, $\pi-\pi$ and Coulombic interaction between IL and dye molecules, and salting-out effect of inorganic salt. Hydrophobicity of the phases makes the main difference among the studied IL-ABSs, and consequently it has considerable impact on dye distribution between phases. The distribution coefficient in [eeim] $]^{+}$based ABSs is generally lower in comparison to [bbim $]^{+}$aqueous systems (Fig. 4b). If we focuse on the biphasic region where the extraction point lay (Fig. S2 $\dagger$ ), it is obvious that salting-out effect dominates in the case of [bbim][dca] because the extraction point is far from its solubility curve. It is likely the reason behind the highest $D$ value for both dyes in the ABS based on [bbim][dca]. 
Additionally, the distribution ratio values of OII (417.2) and RBBR (282.7) for the [heim][Br] ABS are similar to [bbim][Br] ABS. On the other hand, lower D for OII (584.3) and RBBR (316.8) were obtained in the ABS based on [heim][dca] comparing to structural isomer [bbim][dca] indicating their similar behavior.

The calculated binding energies of IL-cation/IL-anion and IL-cation/dye interactions (Table 2) as well as optimized geometries with non-covalent interactions (Fig. S4 $\dagger$ ), were engaged in order to explain the distribution of dyes in the IL-ABSs. The more negative $\Delta G_{\text {bin }}$ values and a higher number of non-covalent interactions were obtained for the interactions between symmetrical IL cations and OII in comparison with RBBR. Considering that the molar volume of OII is lower, it can easily approach to IL-cation and establish stronger noncovalent interactions than RBBR which readily explain generally higher D values of OII. On the other side, asymmetricity of $[\text { heim }]^{+}$cation causes weaker interaction between both IL constituents as well as cation-dye and consequently lower distribution coefficient. Also, the less negative $\Delta G_{\text {bin }}$ values for cation and anion of ILs comparing to $\Delta G_{\text {bin }}$ for cations and dyes could be attributed to spontaneously exchange of ILs anions with dyes.

\section{Optimization of the dye extraction}

With respect to the potential design of industrial scale process, the planning and adjusting the extraction conditions and composition of ABS are crucial steps in order to achieve maximal efficiency with minimal consumption of ABS constituents, IL and inorganic salt. This can significantly reduce the costs and contribute to the feasibility to scale-up the process. Based on the extraction features of the studied ABSs, [bbim][dca] is selected as the most promising IL for further process optimization. When considering the widespread application of ABS for wastewater treatment, it should be kept in mind that the ABS components will inevitably enter into water ecosystems through aqueous effluent. Thus, it is of utmost importance to take into account more eco-friendly and biocompatible salting-out species. So, despite of high ability of phosphates to promote ABSs, their usage has some disadvantages from an environmental aspect. Although essential for the growth of plants, an excess of phosphate resulting from outside sources speeds up the eutrophication process and significantly influences the water quality [47]. Due to this fact, an additional step in the wastewater treatment procedure would be required before discharging effluent to the surface water. In this case, increased costs and process complexity would not justify the utilization of ABS for dye removal from industrial wastewaters. However, one of the major advantages of ABSs is emphasized in this situation. By virtue of their tunability, instead of a phosphate salt, the optimization steps were 
performed using sodium citrate, since it is biodegradable and non-toxic for the aquatic environment. Citrates are weaker kosmotropes then $\mathrm{K}_{3} \mathrm{PO}_{4}$, but in the combination with exceptionally hydrophobic [bbim][dca], that can be easily salted-out from the water, the difference in ABS formation is almost negligible (Fig. S5 and Table S3 $\uparrow$ ).

An appropriate manipulation of the phases' properties which control the selectivity and the partition of the solute of interest is always required for the enhanced extraction. After the selection of the most promising IL-ABS $\left\{\left[\right.\right.$ bbim] $[$ dca $\left.]+\mathrm{Na}_{3} \mathrm{C}_{6} \mathrm{H}_{5} \mathrm{O}_{7}+\mathrm{H}_{2} \mathrm{O}\right\}$ the next very important step from the aspect of process optimization is to investigate the change of extraction parameters with the length of TL and along the same TL. This is of particular importance in order to ascertain $\mathrm{ABS}$ to be more economical and environmentally benign by decreasing the concentration of IL and salt without losing the complete extraction efficiency. The dependence of the distribution ratio of the selected dye (OII) on the compositions of the top and bottom phases expressed through TLL is demonstrated in Fig. 5. Tie-line length equals zero when compositions of the phases are the same (critical point of ABS, Fig. S6 and Table S6 †) and distribution coefficient is close to unity. The distribution ratio increases with increasing of the tie line length and reaches a maximum at tie line length of 86 . The main features of $\mathrm{ABS}$ to be used as enrichment and removal platforms comprises the presence of very long tie-lines because of the high enrichment factor that can be achieved in a single step.

Additionally, the ABS mixture composition within the single tie-line influence on the extraction performance (Fig. 6). By varying the concentrations of IL and salt along the tie line (TLL $=86$ ), the volumes of IL-rich and salt-rich phases are changing while total system volume remains constant (Table S7 $\uparrow$ ). In this way, the volumes of the phases can be easily adjusted so that high extraction efficiency can be achieved with optimal usage of IL and salt. From Fig. 6 it is clear that as the volume of IL-phase decrease, D value significantly increases from 250 to 1750 and extraction efficiency is above $99.4 \%$ for all systems. So, the complete extraction of OII was always attained for all the mixture compositions evaluated. The weight ratio between the water (containing OII) added in the mixture and the IL-rich phase ranges from 2 to 11 (Fig. 6). For the mixture point with a concentration factor of 11, it was experimentally possible to concentrate OII up to 1000-fold in one-step regarding its saturation solubility in water $\left(116 \mathrm{~g} \mathrm{~L}^{-1}\right)$. The saturation limit of the IL-rich phase is well above the saturation limit of OII in water meaning that the IL-rich phase can be reused until achieving the maximum allowable concentration of OII in IL-phase (experimentally determined saturation solubility of OII in [bbim][dca] is $239.4 \mathrm{~g} \mathrm{~L}^{-1}$ ). Taking into account the saturation solubility value of OII in the IL-rich phase and a final concentration required for an accurate 
quantification of the dye (constrained by LOD of UV-VIS spectrophotometer, $1 \times 10^{-3} \mathrm{~g} \mathrm{~L}^{-1}$ ), it can be concluded that [bbim][dca]-based ABS is outstanding as promising concentration platform since theoretically OII can be concentrated up to 239400 -fold.

In order to meet green chemistry principles, the recycling of IL after extraction was also considered. Removal of RBBR from IL after the extraction can easily be achieved in three steps that include the addition of activated carbon, shaking for 1 hour and filtration (Fig. 7). Contrary to RBBR, removal of OII has been a task of many studies investigated various physical-chemical processes and biological methods in order to remove and degrade this dye from the aqueous environment $[15,48]$. Some of these methods presumably can be applied to remove OII from IL-rich phase in order to recycle IL. The pre-concentration of OII using ILABS can simplify the application of these complex degradation techniques requiring less amount of chemicals and shorter time to achieve degradation. However, the application and investigation of the complicate OII removal method is beyond the scope of this work.

\section{Conclusion}

In this work the ABS extraction method based on new symmetric ILs ([bbim][dca]), $[\mathrm{bbim}][\mathrm{Br}],[$ eeim] [dca], and [eeim][Br] ) was investigated and optimized for the removal of two commonly used water-soluble but highly toxic textile dyes (Orange II and Remazol brilliant blue R) from the industrial wastewaters. Firstly, the phase diagrams of ILs-ABS were determined and ability of the ILs to pronounce ABS decrease in following order: [bbim][dca] $>[$ bbim $][\mathrm{Br}]>[$ eeim $][\mathrm{dca}]>[$ eeim $][\mathrm{Br}]$. It was shown that $[\mathrm{bbim}][\mathrm{dca}]$ is the most efficient IL that forms ABS with a exceptionally low amount of salt $(\sim 2 \%)$. Also, it is found that symmetry/asymmetry of imidazolium cation impact the ABS formation and alkyl chains from both positions contribute to the overall hydrophobicity of IL: [bbim][dca] has a significantly higher ability to induce ABS than its structural isomer [heim][dca]. The extraction efficiency of the dyes from aqueous solutions reach $\geq 99.4 \%$ applying ABS based on [bbim][dca] i.e. the complete removal of the dyes were obtained in a single-step procedure. In order to assure the process feasibility, optimization of the procedure using eco-friendly and biocompatible sodium citrate salt instead of potassium phosphate was performed. It was showed that by adjusting the extraction conditions and composition of ABS, the maximal efficiency with minimal consumption of the ABS constituents can be achieved. Using the molecular dynamics simulations, the binding energies of IL-cation/IL-anion and IL-cation/dye interactions as well as optimized geometries with non-covalent interactions were calculated. The more negative $\Delta \mathrm{G}_{\text {bin }}$ values and a higher number of non-covalent interactions between 
symmetrical IL cations and OII in comparison with RBBR agree with experimental extraction results. Finally, recycling and re-use of the applied ILs for the extraction process was also proposed by addition of activated carbon, shaking for 1 hour and filtration.

Funding: This work was supported by the Ministry of Education, Science and Technological Development of Serbia under project contracts III 45006 and ON 172012.

The authors have declared no conflict of interest. 


\section{References}

[1] P.T. Anastas, J.C. Warner, Green chemistry : theory and practice, Oxford University Press, 1998.

[2] E. Gurr, Synthetic dyes in biology, medicine and chemistry, Academic Press, 1971.

[3] E. Forgacs, T. Cserháti, G. Oros, Environ. Int. 30 (2004) 953-971. https://doi.org/10.1016/j.envint.2004.02.001.

[4] H. Ali, Water, Air and Soil Pollut. 213 (2010) 251-273. https://doi.org/10.1007/s11270010-0382-4

[5] E. Brillas, C.A. Martínez-Huitle, Appl. Catal. B Environ. 166-167 (2015) 603-643. https://doi.org/10.1016/j.apcatb.2014.11.016.

[6] M.M. Islam, K. Mahmud, O. Faruk, M.S. Billah, Int. J. Environ. Sci. Dev. 2 (2011) 428-436. https://doi.org/10.7763/ijesd.2011.v2.164

[7] Pulse of the fashion industry, https://globalfashionagenda.com/wpcontent/uploads/2017/05/Pulse-of-the-Fashion-Industry_2017.pdf

[8] D. Rawat, V. Mishra, R.S. Sharma, Chemosphere 155 (2016) 591-605. https://doi.org/10.1016/J.CHEMOSPHERE.2016.04.068

[9] R.L. Stingley, W. Zou, T.M. Heinze, H. Chen, C.E. Cerniglia, J. Med. Microbiol. 59 (2010) 108-114. https://doi.org/10.1099/jmm.0.012617-0

[10] The European Parlament and the Council of the European Union, COMMISSION REGULATION (EC) No 552/2009, Off. J. Eur. Union 2009. 164 (2009) 7.

[11] B.J. Brüschweiler, S. Küng, D. Bürgi, L. Muralt, E. Nyfeler, IRegul. Toxicol. Pharmacol. 69(2) (2014) 263-271. https://doi.org/10.1016/J.YRTPH.2014.04.011

[12] P.F. Gordon, P. Gregory, Anthraquinone Dyes, Organic Chemistry in Colour, Springer Berlin Heidelberg, Berlin, Heidelberg, 1987, pp. 163-99.

[13] European commission, Healt and Food Safety, Opinion on "Assessment of the risks to human health posed by certain chemicals in textiles" http://ec.europa.eu/health/scientific_committees/environmental_risks/opinions/sctee/sct _out72_en.htm

[14] F. Report, S. Feinman, P. Officer, U.S.C. Product, S. Commission, E. Control, T.D. Building, Anthraquinone Dye Poxicological Profiles, https://ntrl.ntis.gov/NTRL/dashboard/searchResults/titleDetail/PB83166033.xhtml

[15] T. Ohashi, A. Jara, A. Batista, L. Franco, M. Lima, M. Benachour, C. da Silva, G. $\begin{array}{lllll}\text { Campos-Takaki, } & \text { Molecules } & 17 & \text { (2012) }\end{array}$ 
https://doi.org/10.3390/molecules171214219

[16] Z. Cai, Y. Sun, W. Liu, F. Pan, P. Sun, J. Fu, Environ. Sci. Pollut. Res. 24 (2017) 15882-15904. https://doi.org/10.1007/s11356-017-9003-8

[17] A. Ayati, M.N. Shahrak, B. Tanhaei, M. Sillanpää, Chemosphere 160 (2016) 30-46. https://doi.org/10.1016/j.chemosphere.2016.06.065

[18] F.A. Kuleyin, Environ. Prog. Sustain. Energy $30 \quad$ (2011) 141-151. https://doi.org/10.1002/ep

[19] Z. Huang, Z. Zeng, A. Chen, G. Zeng, R. Xiao, P. Xu, K. He, Z. Song, L. Hu, M. Peng, T. Huang, G. Chen, Chemosphere $203 \quad$ (2018) 199-208. https://doi.org/10.1016/jchemosphere.2018.03.144

[20] Z. Huang, G. Chen, G. Zeng, Z. Guo, K. He, J. Hazard. Mater. 321 (2017) 37-46. https://doi.org/10.1106/j.jhazmat.2016.08.075

[21] T. Welton, Chem Rev. 99 (1999) 2071-2084. https://doi.org/10.1021/cr980032t CCC:

[22] M. Koel, Crit. Rev. Anal. Chem. 35 (2005) 177-192. https://doi.org/10.1080/10408340500304016

[23] R.D. Rogers, K.R. Seddon, Science $302 \quad$ (2003) 792-793. https://doi.org/10.1126/science.1090313

[24] J.D. Holbrey, Ionic Liquids, Clean Technol. Environ. Policy 1 (1999) 223-236. https://doi.org/10.1007/s100980050036

[25] N. Meksi, A. Moussa, J. Clean. Prod. 161 (2017) 105-126. https://doi.org/10.1016/j.jclepro.2017.05.066

[26] M.G. Freire, Ionic-Liquid-Based Aqueous Biphasic Systems, Springer Berlin Heidelberg, Berlin, Heidelberg, 2016.

[27] J.H. Santos, F.A.E Silva, S.P.M. Ventura, J.A.P. Coutinho, R.L. de Souza, C.M.F. Soares, Á.S. Lima, Biotechnol. Prog. $31 \quad$ (2015) 70-77. https://doi.org/10.1002/btpr.2000

[28] H. Passos, D.J.P. Tavares, A.M. Ferreira, M.G. Freire, J.A.P. Coutinho, ACS Sustain. Chem. Eng. 4 (2016) 2881-2886. https://doi.org/10.1021/acssuschemeng.6b00485

[29] M.G. Freire, A.F.M. Cláudio, J.M.M. Araújo, J.A.. P. Coutinho, I.M. Marrucho, J.N.C. Lopes, L.P.N. Rebelo, Chem. Soc. Rev. 41 (2012) 4966-4995. https://doi.org/10.1007/s00216-017-0705-z

[30] T. Trtić-Petrović, A. Dimitrijević, N. Zdolšek, J. Đorđević, A. Tot, M. Vraneš, S. Gadžurić, Anal. Bioanal. Chem. 410 (2017) 155-166. https://doi.org/10.1007/s00216017-0705-z 
[31] A.M. Ferreira, P.D.O. Esteves, I. Boal-Palheiros, A.B. Pereiro, L.P.N. Rebelo, M.G. Freire, Green Chem. 18 (2016) 1070-1079. https://doi.org/10.1007/s00216-017-0705-z

[32] A.F.M. Claudio, M.G. Freire, C.S.R. Freire, A.J.D. Silvestre, J.A.P. Coutinho, Sep. Purif. Technol. 75 (2010) 39-47. https://doi.org/10.1007/s00216-017-0705-z

[33] S.P.M. Ventura, C.M.S.S. Neves, M.G. Freire, I.M. Marrucho, J. Oliveira, A.P. Coutinho, J. Phys. Chem. B 113 (2009) 5194-5199. https://doi.org/10.1007/s00216017-0705-Z

[34] S.P.M. Ventura, C.M.S.S. Neves, M.G. Freire, I.M. Marrucho, J. Oliveira, J.A.P. Coutinho, J. Phys. Chem. B 113 (2009) 9304-9310. https://doi.org/10.1007/s00216017-0705-Z

[35] A. Dimitrijević, N. Zec, N. Zdolšek, S. Dožić, A. Tot, S. Gadžurić, M. Vraneš, T. Trtić-Petrović, J. Ind. Eng. Chem. 40 (2016) 152-160. https://doi.org/10.1021/je200714h

[36] A. Dimitrijevic, T. Trtić-Petrović, M. Vraneš, S. Papović, A. Tot, S. Dožić, S. Gadžuric, J. Chem. Eng. Data 61 (2016) 549-555. https://doi.org/10.1021/je200714h

[37] Y. Deng, T. Long, D. Zhang, J. Chen, S. Gan, J. Chem. Eng. Data 54 (2009) 24702473. https://doi.org/10.1021/je200714h

[38] S.P.M. Ventura, S.G. Sousa, L.S. Serafim, Á.S. Lima, M.G. Freire, J.A.P. Coutinho, J. Chem. Eng. Data 56 (2011) 4253-4260. https://doi.org/10.1021/je200714h

[39] J.C. Merchuk, B.A. Andrews, J.A. Asenjo, J. Chromatogr. B 711 (1998) 285-293. https://doi.org/10.1016/s0378-4347(97)00594-X

[40] E.R. Johnson, S. Keinan, P. Mori Sánchez, J. Contreras García, A.J. Cohen, W. Yang, J. Am. Chem. Soc. 132 (2010) 6498-6506. https://doi.org/10.1021/jp300874u

[41] S.F. Boys and F.Bernardi, Mol. Phys. 19 (1970) 553-556.

[42] S. Shahriari, C.M.S.S. Neves, M.G. Freire, J.A.P. Coutinho, J. Phys. Chem. B 116 (2012) 7252-7258. https://doi.org/10.1021/jp300874u

[43] M.A.A. Rocha, C.M.S.S. Neves, M.G. Freire, O. Russina, A. Triolo, J.A.P. Coutinho, L.M.N.B.F. Santos, J. Phys. Chem. B 117 (2013) 10889-10897. https://doi.org/10.1021/jp406374a

[44] A.M. Fernandes, M.A.A. Rocha, M.G. Freire, I.M. Marrucho, J.A.P. Coutinho, L.M.N.B.F. Santos, J. Phys. Chem. B $115 \quad$ (2011) 4033-4041. https://doi.org/10.1021/jp201084x

[45] N.J. Bridges, K.E. Gutowski, R.D. Rogers, Green Chem. 9 (2007) 177-183. https://doi.org/ 10.1039/b611628k 
[46] M.H. Ghatee, A.R. Zolghadr, Fluid Phase Equilib. 263(2) (2008) 168. https://doi.org/

[47] D.L. Correll, J. Environ. Qual. 27 (1998) 261-266.

[48] B. Bakheet, S. Yuan, Z. Li, H. Wang, J. Zuo, S. Komarneni, Y. Wang, Water Res. 47 (2013) 6234-6243. https://doi.org/10.1016/j.watres.2013.07.042 


\section{Figure captions}

Fig. 1. Structures of the synthetic dyes (a) OII and (b) RBBR.

(a)

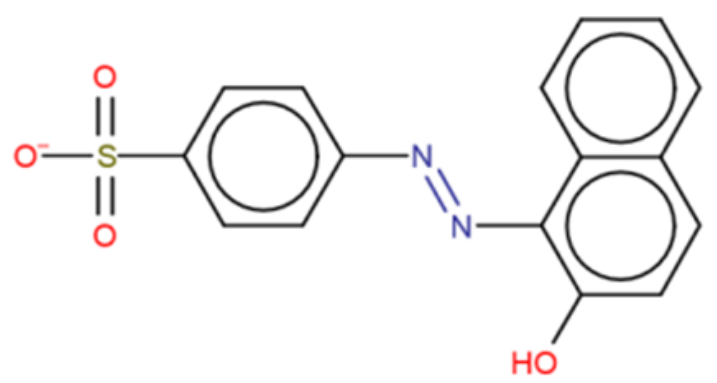

(b)

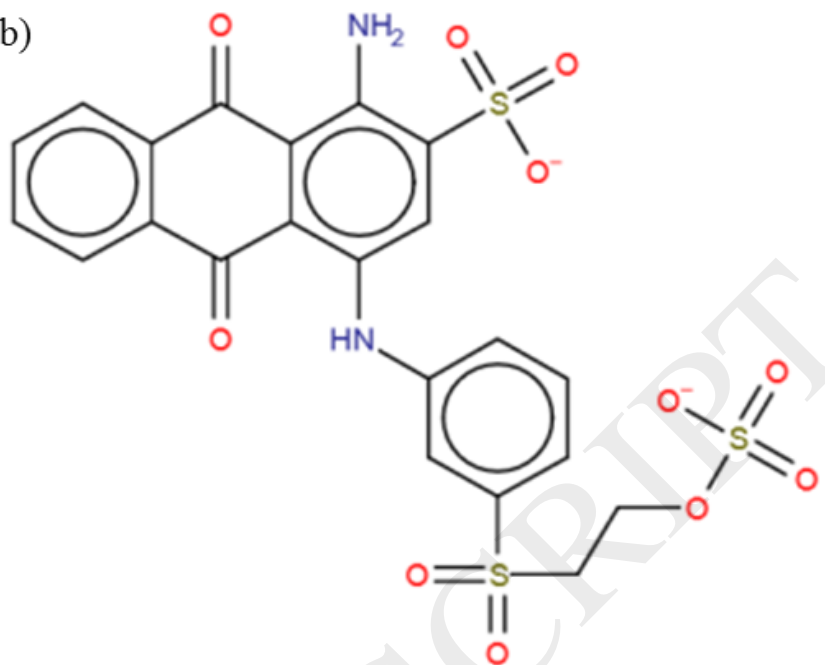

Fig. 2. Ternary phase diagrams of the studied $\left\{\mathrm{IL}+\mathrm{K}_{3} \mathrm{PO}_{4}+\mathrm{H}_{2} \mathrm{O}\right\}$ systems based on the symmetric ILs at $296.15 \mathrm{~K}$ and $0.1 \mathrm{MPa}$. Legend: $\boldsymbol{\Delta}$ [bbim][dca], $\boldsymbol{\nabla}$ [bbim][Br], [eeim][dca] and $>$ [eeim][Br]. Inset: Comparison of binodal curves based on the symmetric and asymmetric ILs with the same number of total methylene groups in the alkyl side chains. Legend: $\diamond[$ heim $][$ dca $]$ and $\square[$ heim $][\mathrm{Br}]$. The experimental solubility data for the ABSs based on [heim][dca] and [heim] $[\mathrm{Br}]$ are given in Table $\mathrm{S} 3 \uparrow$ and taken from our previous paper[35], respectively. 


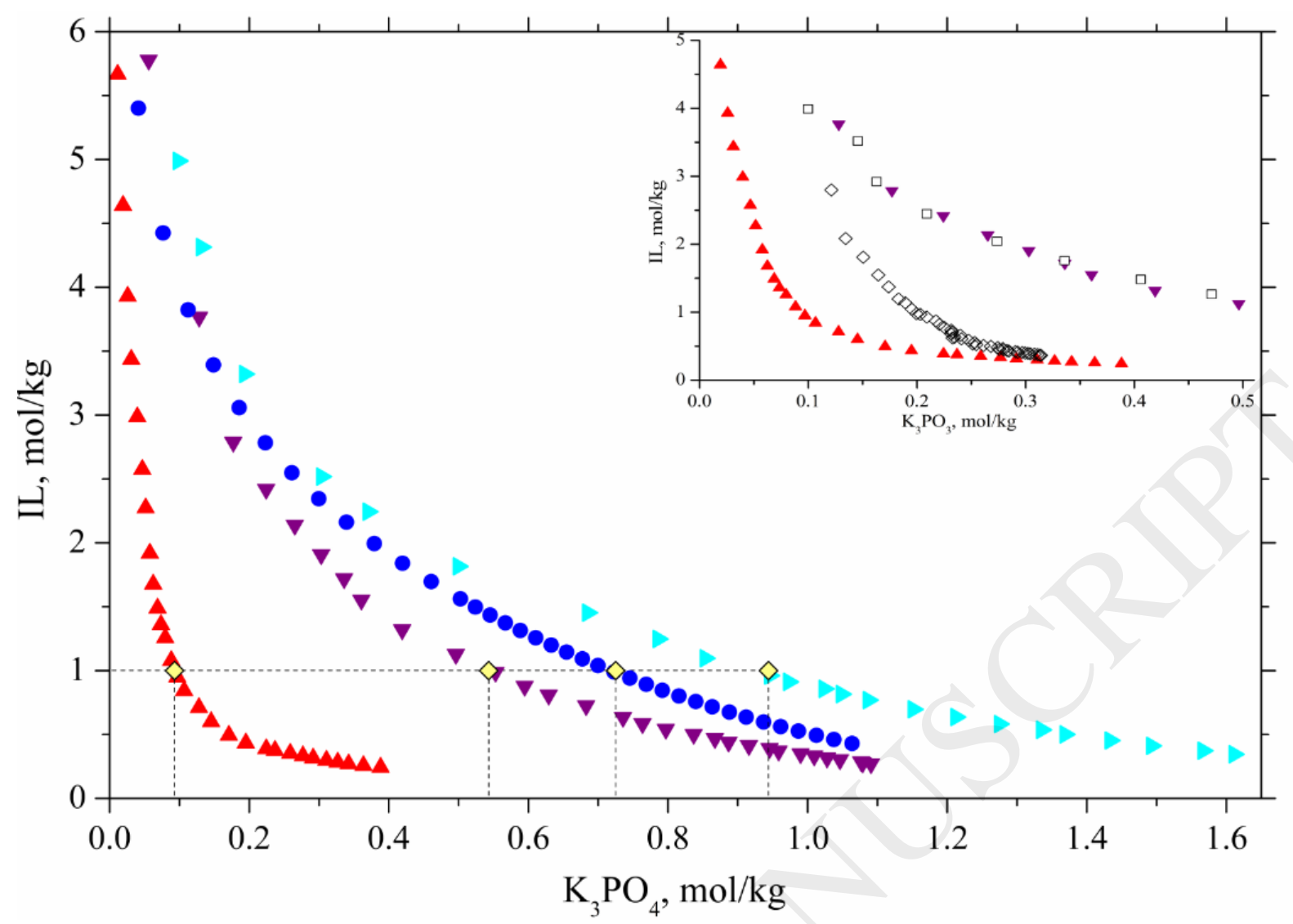

Fig. 3. The calculated values of binding energies between cation and anion of the studied ILs.

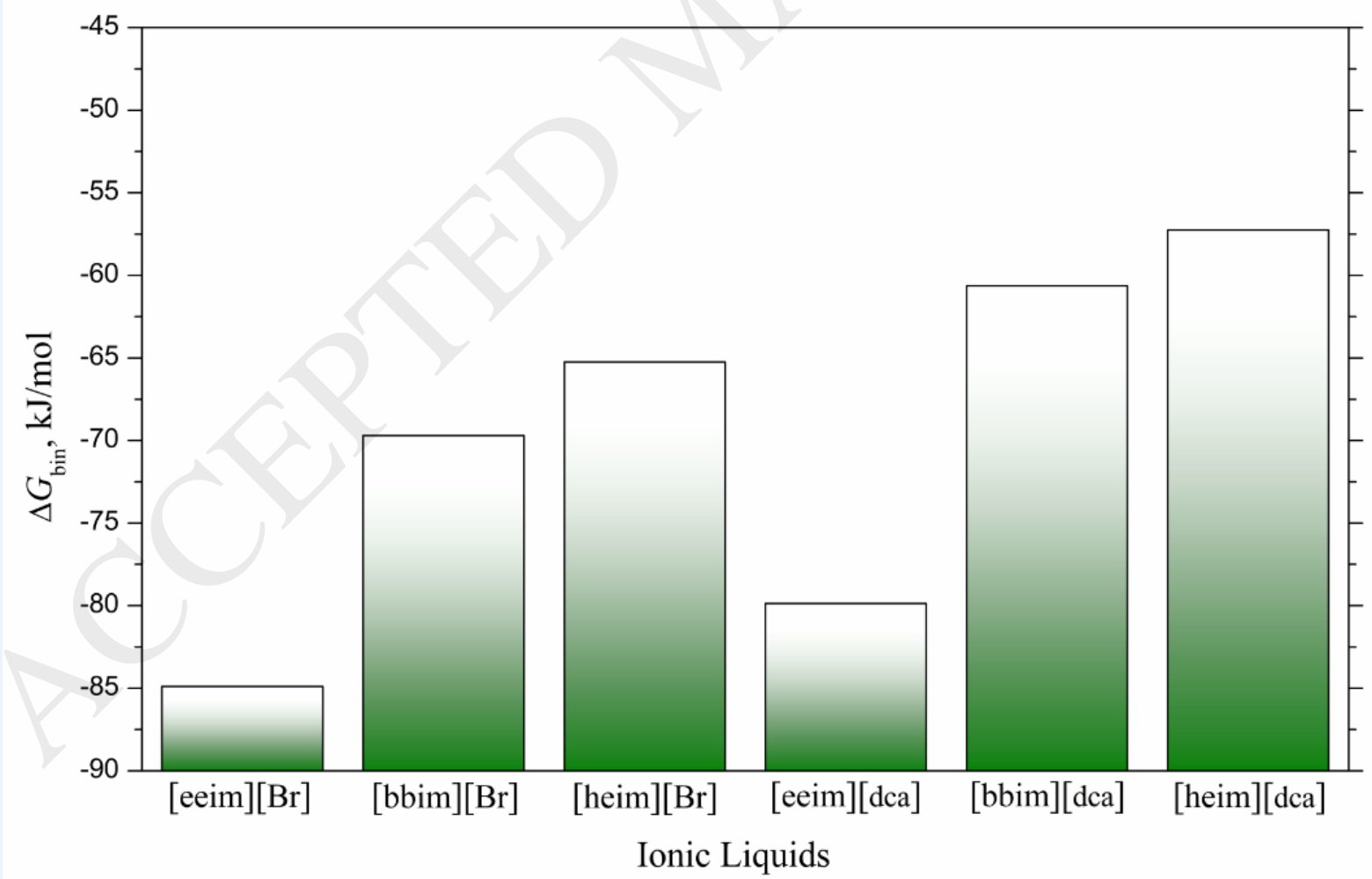


Fig. 4. (a) Picture of the IL-ABS based dye extraction; (b) distribution ratio (column) and extraction efficiencies (line + symbol) of OII (orange) and RBBR (blue) dyes in $\left\{\mathrm{IL}+\mathrm{K}_{3} \mathrm{PO}_{4}\right.$ $\left.+\mathrm{H}_{2} \mathrm{O}\right\}$ ABS.
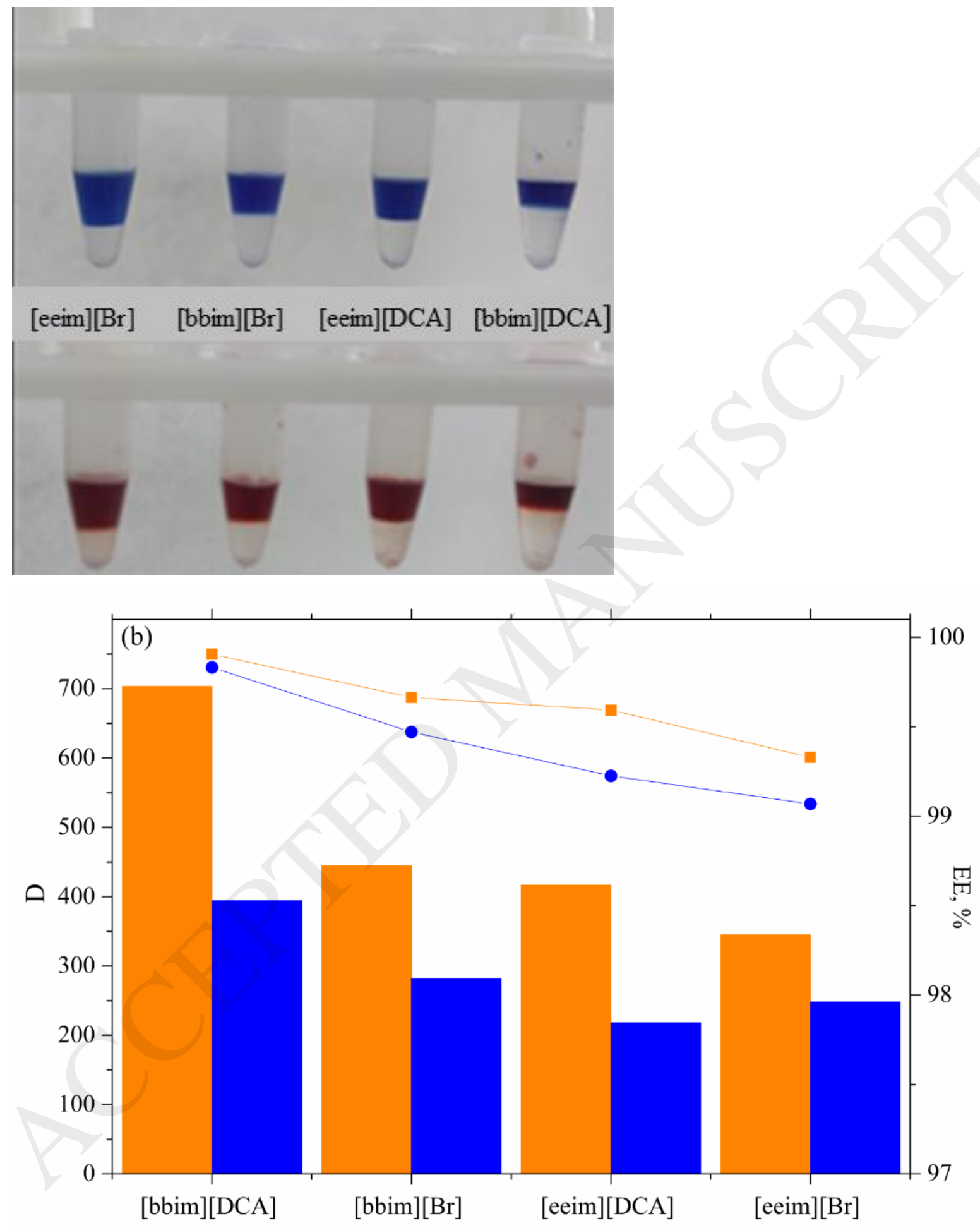
Fig. 5. The phase diagram of $\left\{[\right.$ bbim $][$ dca $\left.]+\mathrm{Na}_{3} \mathrm{C}_{6} \mathrm{H}_{5} \mathrm{O}_{7}+\mathrm{H}_{2} \mathrm{O}\right\}$ ABS (o); TL ( $\boldsymbol{\Delta}$ ); mixture compositions ( $\diamond$; values of TLL are display in gray rectangle and critical point $(\diamond)$. Inset: the dependence of distribution ratio of OII on TLL ( $\mathbf{\square})$.

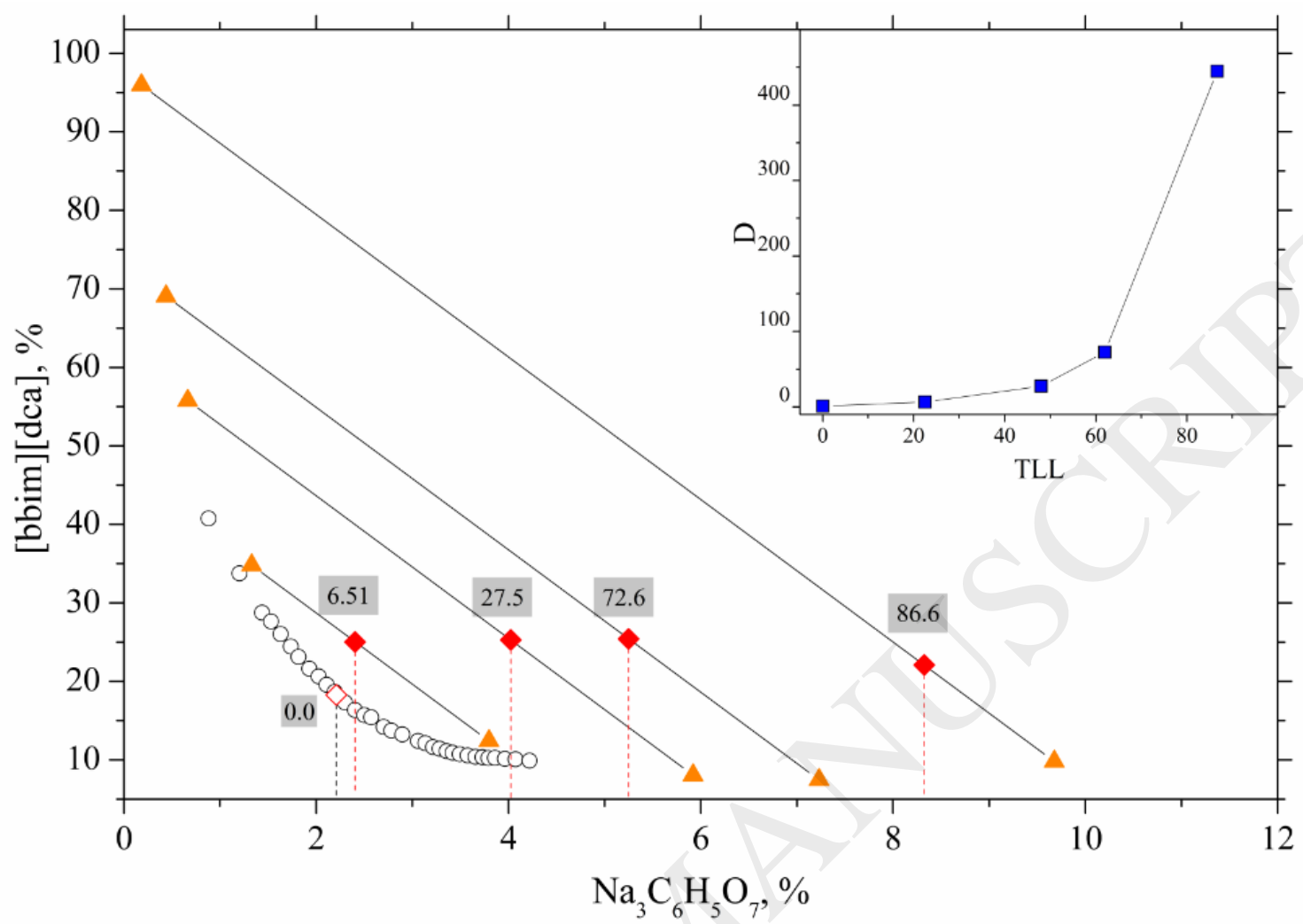

Fig. 6. The effect of phase volumes of ABS on the partition coefficient of OII. Inset: The phase diagram of [bbim] [dca] / $\mathrm{Na}_{3} \mathrm{C}_{6} \mathrm{H}_{5} \mathrm{O}_{7}$ based ABS (o); TL ( $\boldsymbol{\Delta}$ ) and ABS mixture compositions $(\diamond)$. 


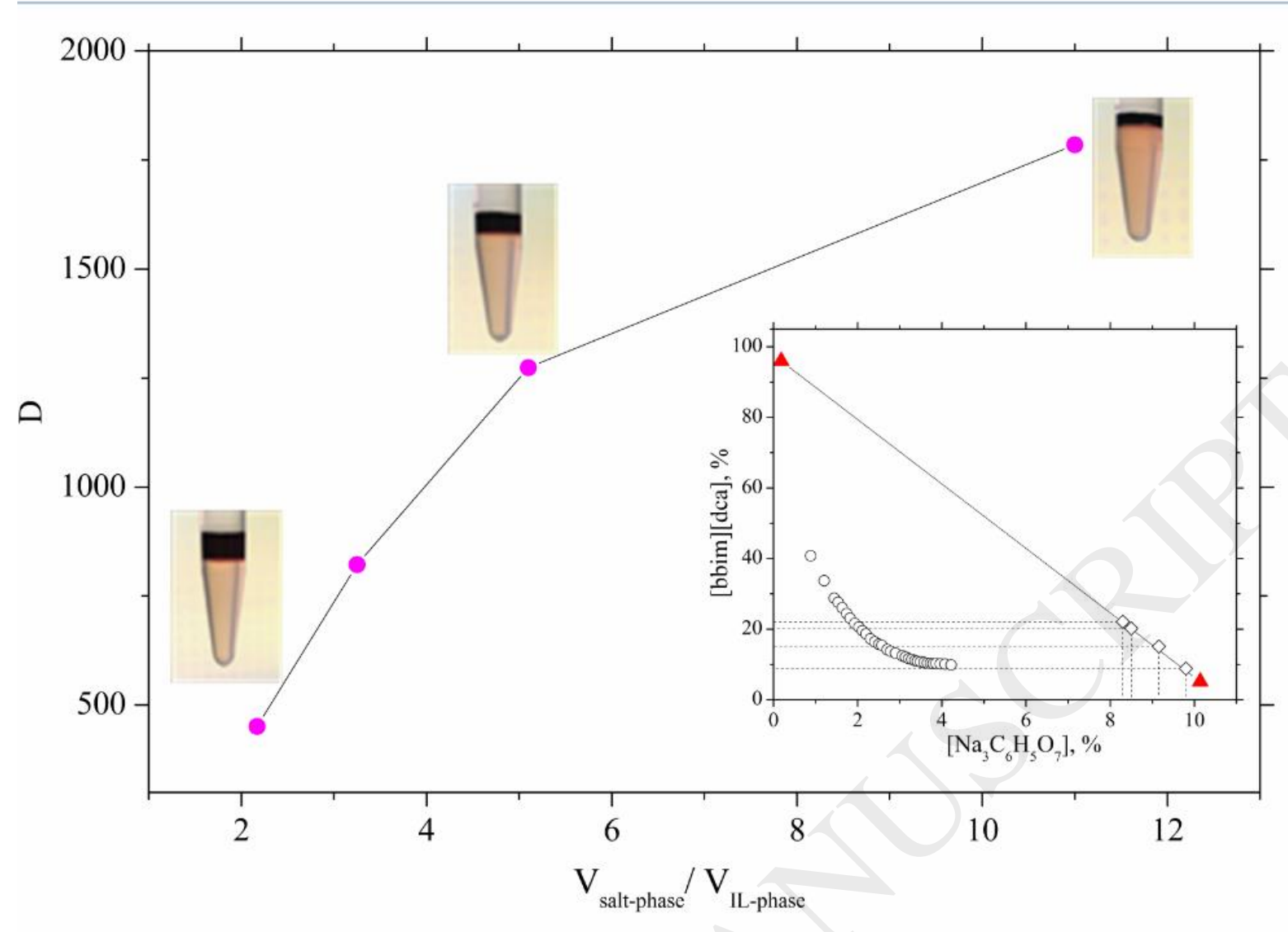

Fig. 7. Recycling procedure of [bbim][dca] (top phase).
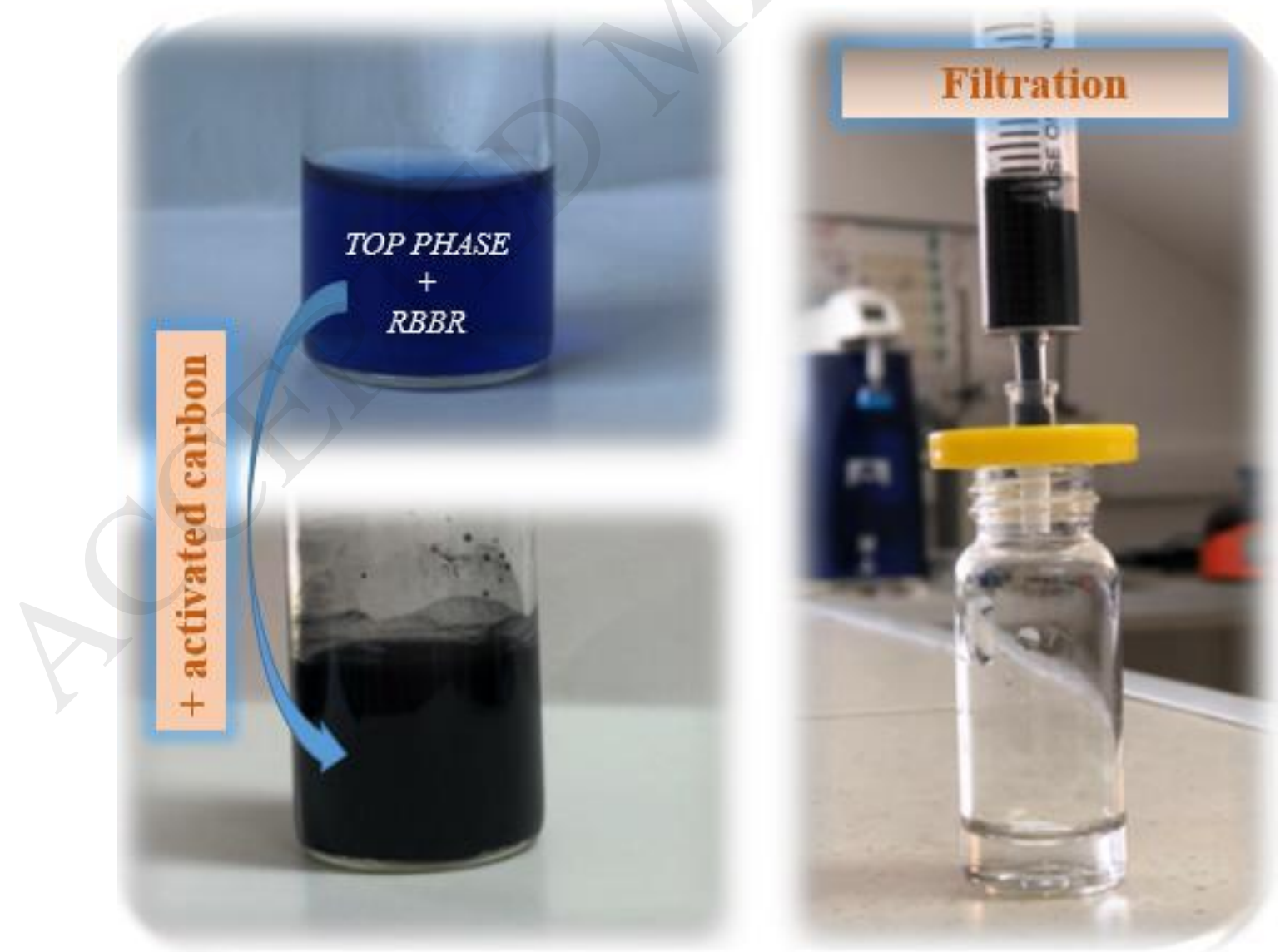
Table 1 The chemical structures and purities of the studied ILs

\begin{tabular}{|l|l|l|}
\hline Ionic Liquid & Chemical structure & $\begin{array}{l}\text { Final } \\
\text { mass fraction }\end{array}$ \\
\hline$[$ eeim] $[\mathrm{Br}]$ & {$[$ eeim][dca] } & $\omega>0.98$ \\
\hline$[$ bbim] $[\mathrm{Br}]$ & {$[$ bbim][dca] } & {[} \\
\hline
\end{tabular}


Table 2 Binding energies $\left(\Delta G_{\text {bin }}\right)$ and number of non-covalent interactions between IL-cation / IL-anion and the IL-cation / dye

\begin{tabular}{|l|l|l|l|l|}
\hline \multirow{2}{*}{ Cation } & \multicolumn{4}{|l|}{$\Delta G_{\text {bin }}, \mathrm{kJ} \cdot \mathrm{mol}^{-1} /$ Number of non-covalent interactions } \\
\cline { 2 - 5 } & {$[\mathrm{Br}]^{-}$} & {$[\mathrm{dca}]^{-}$} & {$[\mathrm{OII}]$} & {$[\mathrm{RBBR}]$} \\
\hline$[\text { eeim }]^{+}$ & $-85.03 / 4$ & $-79.87 / 4$ & $-98.27 / 8$ & $-88.26 / 10$ \\
\hline [bbim $^{+}$ & $-69.86 / 5$ & $-60.63 / 6$ & $-113.3 / 14$ & $-95.43 / 8$ \\
\hline$[\text { heim }]^{+}$ & $-65.25 / 6$ & $-57.25 / 6$ & $-93.41 / 5$ & $-100.9 / 15$ \\
\hline
\end{tabular}

